\title{
Estructuras de Educación Virtual en la Organización Universitaria. Un Acercamiento a la Sociedad del Conocimiento
}

\author{
Jordy Micheli y Sara Armendáriz \\ Universidad Autónoma Metropolitana, Oficina de Educación Virtual, Av. San Pablo 180, \\ Colonia Reynosa Tamaulipas , Azcapotzalco, México DF -México \\ (e-mail: jordy.micheli@gmail.com ; sara.armendariz@gmail.com)
}

Recibido Jun. 06, 2011; Aceptado Jun. 29, 2011; Versión final recibida Ago. 22, 2011

\begin{abstract}
Resumen
Se presenta un estudio sobre las organizaciones emergentes de educación virtual dentro de universidades latinoamericanas. Se aplicaron 41 cuestionarios a responsables de las Estructuras de Educación Virtual. Se observan las características de los responsables de las estructuras, la trayectoria evolutiva de las mismas y las condiciones básicas de trabajo de los docentes bajo modalidad virtual. Se dan detalles de las funciones de las estructuras de educación virtual y sus responsables y se concluye que son organizaciones emergentes multidisciplinarias y con varias funciones dentro de la universidad pero ubicadas también como actores de la sociedad del conocimiento. Se genera una tipología de los modos de evolución de las estructuras y se muestra un cuadro básico de condiciones de trabajo docente dentro de la modalidad virtual.
\end{abstract}

\section{Virtual Education Structures in University Organization. An Approach to the Knowledge Society}

\begin{abstract}
A study of emergent virtual education organizations within Latin American universities is presented. For this 41 questionnaires that were completed by people in charge of the Structures of Virtual Education. The questioning was guided by the characteristics of those responsible for the organizations, their evolutionary trajectory and the basic working conditions of the virtual education of the teachers. A more detailed view of the virtual education structures functions and those responsible for them are provided concluding that they are multidisciplinary and multi-task emerging organizations within universities but they are also part of the knowledge society. A typology for the way in which the evolution of these structures takes place is generated and a basic picture of the teaching work conditions within virtual modes is presented.
\end{abstract}




\section{INTRODUCCION}

La implantación organizacional de la educación virtual en las universidades es un tema que no ha pasado inadvertido desde los años en que empezó a despuntar esta práctica, por ejemplo Bates (1999) señala la importancia de considerar nuevas estructuras organizacionales para desarrollar una estrategia exitosa de uso nuevas tecnologías para la educación universitaria. Distintas interpretaciones posteriores han manifestado la misma preocupación por vincular el desarrollo educativo bajo el nuevo paradigma de tecnologías digitales, con el ámbito de la organización universitaria y sus necesarias adaptaciones. En sentido general, el fenómeno que nos interesa es la capacidad de aprendizaje institucional y la innovación organizacional para poder generar un nuevo servicio educativo altamente tecnificado y a la par, íntimamente vinculado a los factores sociales de cada universidad.

Para abordar el tema, proponemos, una mirada hacia diferentes características de tipo organizacional acerca de la educación virtual universitaria, aportando datos y una interpretación al respecto, a partir de la información empírica que nos ha brindado una encuesta en 41 universidades latinoamericanas, una región en la cual la educación virtual vive desde los años noventas del siglo pasado una rápida expansión caracterizada por la incorporación de instituciones universitarias tradicionales a las nuevas prácticas basada en tecnología digital (Rama, 2010).

Con las nuevas estructuras organizativas de educación virtual dentro de las universidades, éstas cumplen sus nuevos compromisos en el contexto de múltiples demandas que recaen sobre ellas en esta era conocida como la sociedad del conocimiento, la cual está caracterizada por la aceleración de la producción de conocimiento, el crecimiento del capital intangible para fines productivos, el aumento de la actividad de innovación y la revolución de los instrumentos de conocimiento basados en tecnología digital (David y Foray, 2002). En la sociedad del conocimiento, los actores sociales demandantes de la educación son crecientemente los nativos digitales (Rodriguez, 2010), personas cuya estructuras cognitivas son representativas de lo que Dohuei (2010 ) y Simone (2001) han definido como una nueva fase en el modo de conocer, basado en desarrollos mentales integrados a lo digital. Las computadoras utilizadas con fines educacionales, afirma Sancho- Gil (2006), causan el mismo efecto que en otros campos de la vida social bajo el paradigma de la tecnología digital: modifican el qué y el cómo se piensa, de modo que cambian la estructura de intereses, el carácter de los símbolos y el carácter social del espacio en el cual este proceso tiene lugar.

La educación virtual aparece como proceso innovador coincidiendo con la inclusión generalizada de los jóvenes a los sistemas de enseñanza, durante las dos últimas décadas del siglo pasado. Para Esteve se trata de una transformación radical de las demandas sobre estructuras de enseñanza al nivel secundario (Esteve 2001) y para Sörlin y Vessuri (2007) se trata del "siglo de las universidades ". Las universidades dentro de la sociedad del conocimiento tiene múltiples propósitos dentro de su "compromiso" y entre ellos están la competitividad, el acceso, la descentralización y la producción de bienes públicos (Singh, 2007). La UNESCO (2005) recoge este fenómeno y deposita un reto para los procesos educativos basados en la tecnología digital: deben apoyar el enriquecimiento de los medios de aprendizaje para hacer frente a necesidades del acceso universal.

En su estudio sobre la expansión de una "revolución virtual "en Estados Unidos, Kriger (2001) aporta una tipología de las transformaciones en el espectro educativo superior de ese país y muestra los riesgos de una tendencia novedosa basada en la estandarización de los cursos y la masificación del consumo educativo como un servicio para "usuarios", en detrimento de la interacción social del aprendizaje. La tipología muestra que la irrupción de las tecnologías de información y comunicación (TIC) como instrumento de desarrollo de las funciones educativas universitarias ha permitido generar una diferencia entre universidades tradicionales que mantienen la presencia física del proceso de enseñanza- aprendizaje pero refuerzan su quehacer con la educación virtual, y las que generan dicho proceso íntegramente de modo virtual . Barajas (2002) señala que las innovaciones relevantes se dan en las universidades convencionales que adoptan los sistemas de educación virtual y que es el ambiente externo a las mismas el factor más 
importante para explicar sus cambios internos. Un estudio de la OECD (2005) realizado sobre 19 instituciones universitarias de 13 países que habían adoptado el proceso de educación virtual, matiza el impacto real de la misma sobre los fundamentos educacionales y señala el que se trata aún de un proceso inmaduro que deberá mostrar los efectos sobre el tema educativo a futuro. En dicho estudio se apunta que los objetivos importantes que las universidades persiguen son la flexibilidad en la "entrega "del servicio educativo y el fortalecimiento de la pedagogía, y quedan en un segundo plano de importancia la ampliación de la oferta y la reducción de costos.

Retomamos la referencia a Barajas (2002) puesto que en su estudio sobre 9 universidades europeas incursiona en el análisis del modo en que la implementación de sistemas virtuales de educación se interrelaciona con los cambios organizacionales al nivel institucional. El reto básico, señala el autor, es desarrollar educación virtual dentro de estructuras que pertenecen a viejos modelos organizacionales y a culturas institucionales arraigadas. El autor no va más allá en el análisis organizacional propiamente dicho, pero realiza valiosas observaciones. Una es la existencia de una trayectoria evolutiva de la educación virtual que va desde las iniciativas personales y a nivel piloto hacia una adopción consciente por parte de la institución, dando lugar a tres fases: inicio, implementación e institucionalización. Otra es la necesidad de conocimientos multidiciplinarios entre los actores que deben llevar a cabo la educación virtual, aunque no diferencia entre miembros del personal académico y los responsables de la gestión de la educación virtual.

Garrison y Anderson (2005) plantean que las universidades deben expandir la educación virtual a partir de un proceso de innovación estratégica y de un liderazgo académico de actores claves dentro de la universidad. Los mismos autores resaltan la importancia de los aspectos organizativos mediante los cuales las instituciones educativas se posicionan respecto a la educación virtual: las instituciones se enfrentan al reto de desarrollar una visión y una dirección estratégica que las sitúe en la posición adecuada para avanzar sin reducir la agilidad para adaptarse a nuevos cambios; para responder a este reto, apelan a un liderazgo organizativo dotado de intuición y también de recursos.

Una visión amplia que pretende mostrar qué aspectos son los determinantes para el cambio organizacional y la adopción decisiva de la educación virtual es la que brinda Serbin (2003). Su punto de vista es el de la economía de servicios y el papel de los actores comerciales como propulsores de innovaciones en las universidades. Es un trabajo interesante por su perspectiva económica que mantiene una demanda similar a la otros autores en la parte organizacional: las universidades deben adoptar un cambio organizacional, pero no aporta una explicación de cómo ocurre tal cambio. Nuestra investigación retoma la visión de los autores anteriores, la cual podríamos reformular así: las universidades que adoptan la práctica de la educación virtual bajo cualquiera de sus modalidades, agregan algo más que un instrumento de índole tecnológica para beneficio de sus estudiantes y para ampliar su abanico de ofertas educativas. Lo que hacen esas instituciones es adoptar una nueva función universitaria que reclama algún modo de innovación organizacional, y que ello ocurre bajo las condiciones de la sociedad del conocimiento.

\section{EDUCACION VIRTUAL UNIVERSITARIA}

La educación virtual (EV, en lo sucesivo) es el proceso de enseñanza-aprendizaje que tiene lugar, parcial o totalmente, a través de Internet. Puede ser que el proceso se lleve a cabo sustituyendo totalmente la presencia física del docente (e- learning ) o bien puede ocurrir una combinación de presencia física y trabajo por medio de internet (b-learning). En la práctica universitaria de la EV identificamos, para los fines de nuestro enfoque, a las Estructuras de Educación Virtual (EEV) que son partes dentro de la organización universitaria (facultades, centros, administración, rectorados, etc.), constituidas por grupos de trabajo cuya función es el desarrollo de Sistemas de Educación Virtual (SEV) :estos sistemas son ambientes basados en las tecnologías de información y comunicación (TIC), las cuales son mediadoras entre los estudiantes, los materiales educativos y las actividades docentes y formativas en general (Alavi y Leinder,2003).

Así, las EEV , dentro de las universidades, son las responsables de administrar y expandir el uso de la educación virtual. Es decir, la Estructuras tienen a su cargo a los Sistemas de Educación 
Virtual. Las EEV son encargadas del aprendizaje tecnológico y didáctico en el campo de la EV y de su traducción para la organización universitaria. Ello las diferencia de otras estructuras universitarias: como se sabe las universidades están divididas en diferentes estructuras de generación de conocimiento para los alumnos, y se trata de divisiones que recogen los modos tradicionales de educación e investigación en campos disciplinarios bien constituidos mediante fronteras y paradigmas. Las EEV son también estructuras de generación de conocimiento, pero su objetivo es utilizar y expandir la EV y ello define sus características, de un modo diferente al de las tradicionales de la universidad.

En una interpretación previa (Micheli y Armendáriz, 2005) habíamos visualizado a la EV como una cadena de producción conformada por 4 ámbitos: el tecnológico, el organizacional, el educativo y el del impacto que produce. El primero puede ser visto, desde una perspectiva de servicios, como el espacio de las innovaciones de producto que son adaptadas por el segundo ámbito, el organizacional universitario, para generar innovaciones del proceso de la educación virtual. El tercer ámbito, el educativo, constituye para nosotros el espacio de creación y consumo del servicio educativo, y requiere de las competencias de docentes bajo la modalidad específica en que se organice la entrega del bien educativo. El cuarto ámbito, el del impacto, aborda valores, opiniones, símbolos, etc., que acompañan a la educación virtual como práctica emergente. Esta visión previa nos ha permitido proponer una caracterización de la educación virtual basada en el aprendizaje institucional (Micheli, 2009).

Avanzando con esta propuesta de una mirada estructural, hoy podemos afirmar que la educación virtual es un servicio basado en diversas innovaciones dentro del conjunto de las TIC, por ello puede verse como un proceso educativo en sentido amplio que co-evoluciona con su matriz tecnológica, y con las transformaciones organizacionales como las EEV que hemos mencionado, pero también con nuevos mercados, líneas de innovación, comunidades de expertos y en general con un creciente y complejo sistema de producción de insumos y capacidades para la práctica de educación virtual. La educación virtual ha dejado de ser una práctica periférica del quehacer educativo en general y es ya un campo laboral con importantes agentes innovadores, intereses económicos y un paradigma científico en construcción y diversas vertientes de estudio (Edel, 2009). Las reticencias originales sobre la autenticidad del aprendizaje mediado por la virtualidad, comparado con el aprendizaje bajo la forma tradicional, son cada vez menores y las tendencias de investigación actuales en la educación virtual van dirigidas a reconocer las diferencias existentes entre la educación presencial y la educación en línea, con énfasis en los proceso híbridos y la búsqueda de las mejores formas de lograr la efectividad en la educación con apoyo de Internet (U.S. Department of Education, 2009)

Existen importantes tendencias de innovación dentro del servicio de educación virtual: Una es la de las plataformas de administración del aprendizaje (Paulsen, 2003) y otra es la que se aboca a la producción de objetos de conocimiento (Polsani, 2003). Las primeras han tenido un amplio crecimiento desde su desarrollo en los años noventas del siglo pasado y la denominada Moodle, de uso gratuito, es la más popular y es empleada en más de 54 mil sitios web en el mundo, con 4.5 millones de cursos registrados. Otra plataforma importante es Blackboard, la cual es comercial y es utilizada por cerca de 7 mil organizaciones con fines educativos (la información sobre estas plataformas punteras se puede encontrar en sus correspondientes sitios web). Por su parte, los objetos de conocimiento son bloques de información con diseño instruccional para fines educativos y cuya configuración informática les permite ser interoperables entre diversos sistemas y también reusables.

En ambas líneas de desarrollo se busca una suerte de estandarización de procedimientos con tecnologías aplicadas de uso generalizado, apuntando a escenarios de masificación de la educación virtual. Existe una tercera línea que busca generar experiencias educativas más personales bajo principios de una hibridación de la educación virtual, entre lo presencial y lo virtual. Estas tendencias, y otras que se vislumbran para el futuro mediato e inmediato (García et al. 2010; Wolff et al. 2007) constituyen el núcleo innovador de la educación virtual, en el cual intervienen comunidades, empresas, mercados de TIC, etc. Certeramente a juicio nuestro, Kalay (2004) muestra la importancia de la educación como una actividad socialmente contextualizada y plantea que el reto de la educación virtual universitaria radica en que no debe perder la 
interactividad social, misma que ha caracterizado a la actividad educativa como actividad situada en los campus. Ese reto es tanto de diseño tecnológico como social y forma parte de la estrategia organizativa de la universidad.

Así, desde la perspectiva de un servicio moderno, típico de la sociedad del conocimiento por su contenido en capital humano y por su matriz tecnológica informacional, las EEV constituyen el núcleo productor y responsable de la entrega del servicio educativo, sin olvidar que cada organización es orgánica y única para cada universidad, siguiendo la idea clásica del análisis de organizaciones de Drucker (1997), pero hay elementos comunes en las características del equipo encargado de liderar el proceso, en las vías de su desarrollo y las dinámicas del sistema socio técnico que administra la educación virtual.

\section{EL GRUPO DE UNIVERSIDADES ANALIZADAS}

Nuestra muestra de EEV analizadas comprende 41 casos, que corresponden a otras tantas universidades, como se ve en la Tabla 1. La muestra está basada en una selección de los entrevistados que tuvieran a su cargo EEV, independientemente del lugar jerárquico que ocupara la EEV dentro de la organización universitaria. Se trató de una muestra heterogénea con la finalidad de encontrar los rasgos compartidos, es decir, un muestreo intencional de máxima variación (Izcara, 2007; Hernández, Fernández- Collado, Baptista, 2006).En una primera etapa se hizo una búsqueda de EEV y sus responsables por internet, enviándose 111 cuestionarios en línea y obtuvimos 19 respuestas. La necesidad de mayor información nos motivó a buscar recursos para aplicar el mismo cuestionario de manera presencial. El resultado es que fueron obtenidas 22 entrevistas más de manera directa. El levantamiento de los cuestionarios se realizó durante el año 2010. Obviamente, los casos de entrevistas directas nos ayudaron a contextualizar y obtener información extra para generar una explicación de resultados.

Tabla 1: Lista de universidades y cargos que contestaron nuestro cuestionario

\begin{tabular}{|c|c|}
\hline En línea & Presencial \\
\hline Argentina & Argentina \\
\hline Programa Univ. Virtual Nacional de Quilmes & Univ. de Buenos Aires Facultad de Medicina \\
\hline Director Académico & Secretario de Tecnología Educativa \\
\hline Bolivia & Univ. Tecnológica Nacional Argentina \\
\hline Univ. Privada Boliviana & Subsecretario Académico \\
\hline Coordinador de educación virtual & Ecuador \\
\hline Chile & Escuela Politécnica Nacional Ecuador \\
\hline Univ. de Santiago de Chile & Coordinadora del Centro de Educación Continua CEC \\
\hline Director del Campus Virtual & México \\
\hline Colombia & Univ. de la Salle \\
\hline Univ. INCCA de Colombia & Coordinadora del Centro de Educación a Distancia \\
\hline Director Unidad de Tecnología & Univ. Iberoamericana \\
\hline Ecuador & Dirección de Educación Continua \\
\hline Univ. Nacional de Loja & Coordinadora de Ibero NET. \\
\hline Director de la Modalidad de Estudios a Distancia & Univ. Anáhuac del Sur \\
\hline $\begin{array}{l}\text { Pontificia Univ. Católica del Ecuador Sede Ibarra } \\
\text { Jefe de la Unidad de Educación a Distancia }\end{array}$ & $\begin{array}{l}\text { Coordinador del Centro Corporativo de Educ. en línea } \\
\text { Univ. Virtual de la Anáhuac }\end{array}$ \\
\hline $\begin{array}{l}\text { El Salvador } \\
\text { Univ. Tecnológica de El Salvador }\end{array}$ & Directora de Formación e-learning \\
\hline Coordinador de Educación a Distancia & $\begin{array}{l}\text { Facultad de Estudios Superiores Acatlan UNAM } \\
\text { Jefatura de División del Sistema Univ. Abierta y }\end{array}$ \\
\hline $\begin{array}{l}\text { Guatemala } \\
\text { Univ. Galileo }\end{array}$ & Educación a Distancia \\
\hline $\begin{array}{l}\text { Director de Área de e-learning } \\
\text { Honduras }\end{array}$ & $\begin{array}{l}\text { Univ. Popular Autónoma del Estado de Puebla Online } \\
\text { Directora de la UPAEP Online }\end{array}$ \\
\hline Univ. Nacional Autónoma de Honduras & Univ. Pedagógica Nacional, Campus Puebla \\
\hline Directora de Innovación Educativa & Administrador del Campus Virtual \\
\hline
\end{tabular}


Tabla 1 (continuación)

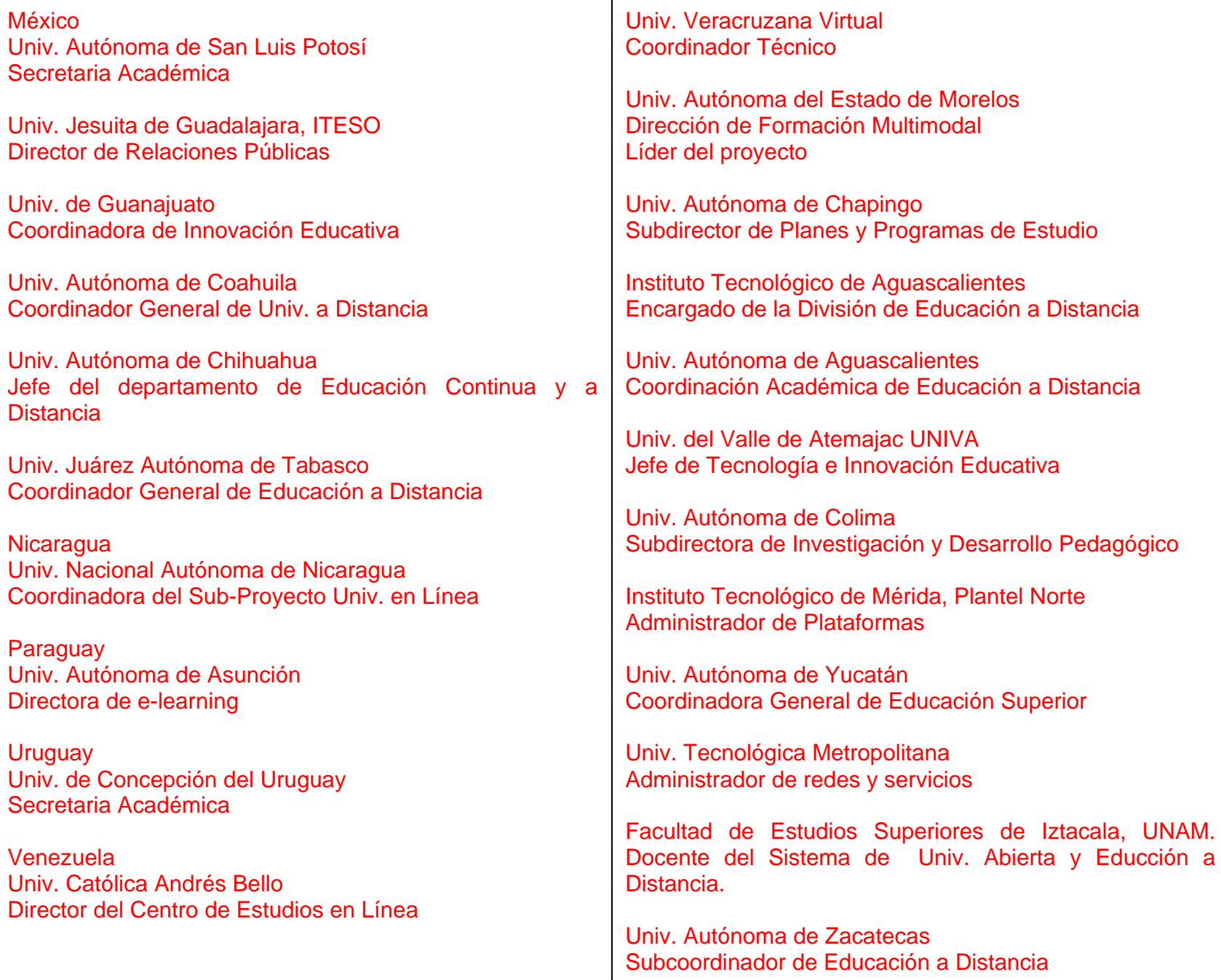

Univ. Veracruzana Virtual

Coordinador Técnico

Univ. Autónoma del Estado de Morelos Dirección de Formación Multimodal Líder del proyecto

Univ. Autónoma de Chapingo

Subdirector de Planes y Programas de Estudio

Instituto Tecnológico de Aguascalientes

Encargado de la División de Educación a Distancia

Univ. Autónoma de Aguascalientes

Coordinación Académica de Educación a Distancia

Univ. del Valle de Atemajac UNIVA

Jefe de Tecnología e Innovación Educativa

Univ. Autónoma de Colima

Subdirectora de Investigación y Desarrollo Pedagógico

Instituto Tecnológico de Mérida, Plantel Norte

Administrador de Plataformas

Univ. Autónoma de Yucatán

Coordinadora General de Educación Superior

Univ. Tecnológica Metropolitana

Administrador de redes y servicios

Facultad de Estudios Superiores de Iztacala, UNAM. Docente del Sistema de Univ. Abierta y Educción a Distancia.

Univ. Autónoma de Zacatecas

Subcoordinador de Educación a Distancia

El cuestionario fue diseñado para su aplicación en línea con preguntas cerradas. Para los encuentros presenciales, el mismo cuestionario fue utilizado como guía de entrevista. Está estructurado de la siguiente manera: una primera parte se dirige a conocer características laborales de la persona a cargo de la EEV; una segunda parte es para caracterizar la EEV en sí misma; y una tercera parte es acerca de las condiciones básicas de la práctica laboral de la educación virtual dentro de la institución.

\section{Resultados}

De las 41 organizaciones bajo estudio, el $59.5 \%$ de ellas fueron creadas durante 2005-2009; $33.5 \%$ durante $2000-2004$ y el restante $7 \%$ de ellas fueron creadas antes del año 2000 . Se trata, pues, de una muestra de universidades con una actividad de educación virtual reciente y no con una actividad consolidada desde el punto de vista de su historia de vida.

Si observamos el tamaño de las EEV desde el punto de vista del número de personas que laboran en ellas, el $35 \%$ de ellas reporta tener entre 1 y 5 personas laborando; 23\%, entre 6 y 10; $24 \%$, entre 11 y 15 y, finalmente, 18\% , 16 o más personas. No encontramos un tamaño claramente dominante,y lo que ello nos sugiere es que aún no se ha podido establecer un patrón de tamaño óptimo para las actividades de educación virtual, situación nada extraña en la medida que se trata de procesos nuevos en instituciones tradicionales. 


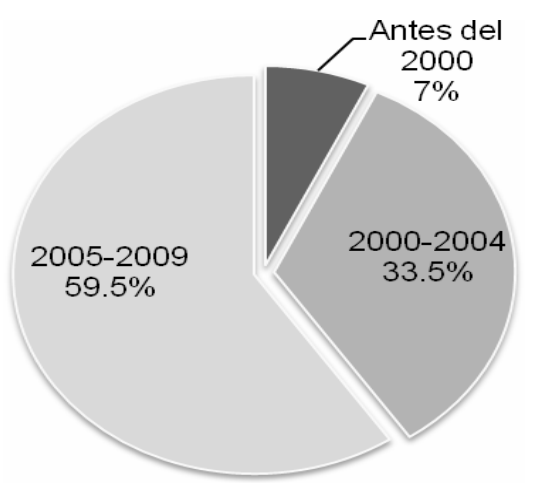

Fig. 1: Periodo de creación de las EEV

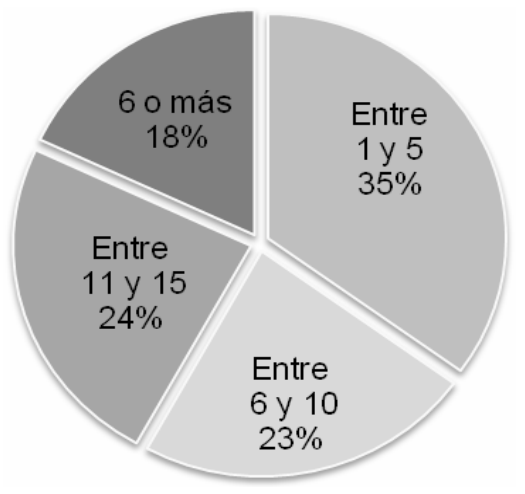

Fig. 2: Tamaño de las EEV por número de personas

Otro dato general que deseamos exponer de inicio es que el $50 \%$ de los entrevistados manifestó que fue la instancia de educación virtual la responsable de elegir la plataforma de aprendizaje (Learning Management System) utilizada en la universidad, y la otra mitad de los entrevistados señaló que fueron las áreas tecnológicas quienes tomaron la decisión. Este es el reflejo de un aspecto interesante en el uso de las TIC dentro de las universidades: la emergencia de la educación virtual puede estar desplazando las decisiones que tradicionalmente han sido potestad de los especialistas tecnológicos.

\section{Características de los responsables de las EEV}

En el personal de dirección de las EEV son predominantes los perfiles laborales multidisciplinarios y una proporción importante de las personas a cargo de esas estructuras tienen un origen disciplinario distinto del campo actual. Ello nos puede sugerir que el desarrollo inicial de estas estructuras siempre implica el aprendizaje de nuevas competencias y conocimientos. De acuerdo con los antecedentes profesionales de la gente a cargo de las EEV, formamos cuatro grupos principales según el campo general de conocimiento: educación, tecnología, otras disciplinas y dos o más disciplinas. Ello se muestra en la Tabla 2. Los objetivos que tienen los responsables de las EEV se muestran en la Tabla 3

Tabla 2: Multidisciplinariedad en las EEV

\begin{tabular}{|l|l|l|}
\hline Campo principal de conocimiento & \% respecto al total & Campos específicos \\
\hline Educación & 35 & Educación, Pedagogía, Sicología \\
\hline Tecnología & 27.5 & $\begin{array}{l}\text { Informática, Sistemas, Computación, } \\
\text { Electrónica, Telecomunicaciones, }\end{array}$ \\
\hline Otras disciplinas & 15 & $\begin{array}{l}\text { Administración, Medicina, Diseño, } \\
\text { Sociología, Ingeniería Mecánica }\end{array}$ \\
\hline Dos o más disciplinas & 22.5 & Educación y Tecnología, Otras \\
\hline
\end{tabular}


Tabla 3: Objetivos de los responsables de EEV

\begin{tabular}{|l|l|}
\hline Objetivo & \% de respuestas \\
\hline Expandir la práctica de la EV en su institución & $92.9 \%$ \\
\hline Realizar investigación y desarrollo & 83.3 \\
\hline Cooperar con otros especialistas & 81.0 \\
\hline Intervención sobre aprendizaje y practica de la EV de los docentes & 66.7 \\
\hline Difusión de las actividades de la EEV & 61.9 \\
\hline Evaluar el efecto de la EV en estudiantes & 61.9 \\
\hline Todas las anteriores & 31.0 \\
\hline
\end{tabular}

Las actividades específicas llevadas a cabo por la gente responsable de las EEV constituyen el "rompecabezas" de la innovación incremental en la EEV para el desarrollo de los SEV: son procesos de aprendizaje organizacional para el uso efectivo de conocimiento adaptado al contexto de las universidades, y abarcan desde ideas puestas en marcha hasta rutinas adaptativas y siempre comprenden trabajo en equipo y/o en redes, como se muestra en la figura 3. Los responsables de la EV perciben un conjunto de responsabilidades son su campo profesional pero enfatizan la expansión de la EV y la cooperación con otros especialistas.

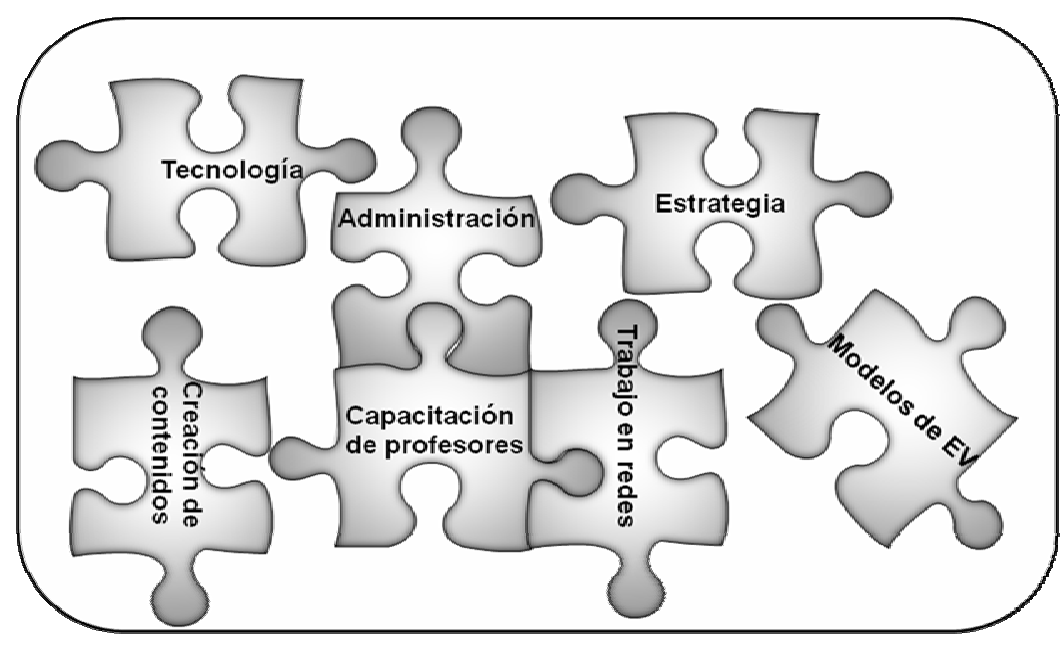

Fig. 3: Actividades de las EEV: un modelo para armar

Los modos de creación y expansión de los SEV y visión de los responsables.

Como mencionamos anteriormente, las EEV que analizamos tienen un período de vida y de experiencias laborales acumuladas, aún cortos. Sin embargo, podemos distinguir formas de creación de dichas estructuras, las cuales son antecedentes que en algún momento futuro podrán ser relacionados con la trayectoria de estas organizaciones universitarias. Les denominamos "modos de creación y expansión de las EEV" y son los siguientes:

- Modo 1: universidades en la cuales la educación a distancia o la educación continua tradicionales determinan la evolución hacia formas de EV.

- Modo 2: universidades en la cuales las formas pioneras de EV fueron desarrolladas por profesores interesados en innovación, y esta práctica focalizada es adoptada en la estructura general de la universidad.

- Modo 3: universidades en las cuales una condición específica, sea una necesidad puntual o bien una ventaja existente, lleva a un proceso oportunista de desarrollo de la EV 
- Modo 4: universidades en las cuales la EV es detonada al más alto nivel de decisión. En estas universidades, la EV generalmente parte de cero.

En los modos 1 y 2 los actores decisivos empujan a la organización, en los modos 3 y 4 la jalan. La proporción de universidades que caen en cada uno de estos 4 modos es la que se muestra a continuación.

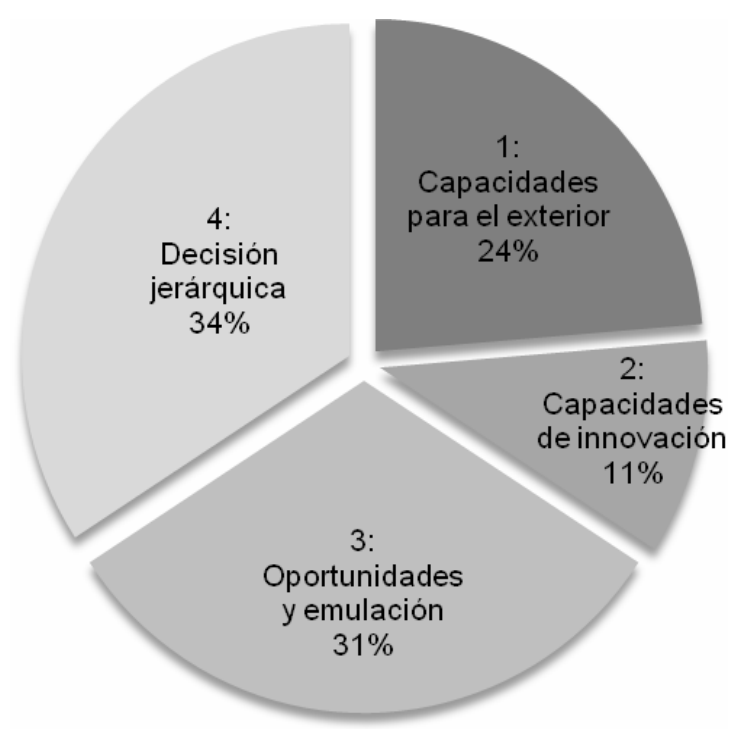

Fig. 4: Modos de expansión de la EV

En relación con la dinámica de las EEV, nos interesó conocer la percepción sobre el estadio de desarrollo en que se encuentra el SEV en cada universidad (fase inicial, de consolidación o consolidada). Así, las repuestas nos indican la distancia entre la situación actual y un futuro posible y/o deseable.

Una característica asociada a la anterior es la referente a la orientación principal que seguirá el desarrollo de la EEV en cada institución: se dirigirá hacia lograr expandir la EV en su universidad, hacia realizar investigación y desarrollo o hacia crear nuevos productos de enseñanza aprendizaje dentro de la EV. El resultado, que se muestra en el cuadro siguiente, pone de relieve que mayormente los SEV se encuentran en fases tempranas y, en consecuencia, las orientaciones básicas son hacia la expansión de la práctica de EV.

Tabla 4: Dinámica de las EEV

\begin{tabular}{|l|l|l|l|l|}
\hline & Inicial & Consolidación & Madura & TOTAL \\
\hline Expansión & 21.2 & 18.2 & 6.1 & $45.5 \%$ \\
\hline Investigación y desarrollo & 12.1 & 12.1 & 9.1 & $33.3 \%$ \\
\hline Nuevos productos & 12.1 & 6.1 & 3.0 & $21.2 \%$ \\
\hline TOTAL & $45.5 \%$ & $36.4 \%$ & $18.2 \%$ & $100.0 \%$ \\
\hline
\end{tabular}

\section{La práctica de los docentes que hacen EV: regulación y voluntariedad}

Al abordar el tema de la práctica de la EV por parte de los docentes, sugerimos que se puede conformar una suerte de "cuadro" básico de posibilidades, formado por el ámbito hacia el cual se dirige la práctica de EV (cursos regulares o educación continua), las normativas que existen para la práctica de la EV, la voluntariedad con la cual se emprenda la EV y el reconocimiento institucional a la práctica de EV. 
Tabla 5: Cuadro básico de la práctica docente de EV

\begin{tabular}{|l|l|}
\hline Educación formal y no formal & Regulación institucional \\
\hline $\begin{array}{l}\text { - cursos regulares universitarios únicamente: } 38 \% \\
\text { - educación continua únicamente: } 15 \%\end{array}$ & $\begin{array}{l}\text { - en el } 60 \% \text { de los casos la EV está sujeta a a } \\
\text { alguna forma de regulación institucional } \\
\text { - en el } 40 \% \text { restante no ocurre tal regulación. }\end{array}$ \\
\hline Participación docente & Reconocimiento institucional \\
\hline $\begin{array}{l}\text { - en el } 76 \% \text { de los casos los docentes hacen } \\
\text { voluntariamente }\end{array}$ & $\begin{array}{l}\text { - en } 59 \% \text { de los casos no hay reconocimiento } \\
\text { - en el lo hay en el } 41 \% \text { restante. }\end{array}$ \\
\hline
\end{tabular}

\section{CONCLUSIONES}

Nuestra investigación abarcó una mayoría de casos en que la creación de EEV es reciente, lo cual se reflejó en los indicadores sobre la caracterización del desarrollo de las mismas. La mayoría lo señaló como en una fase incipiente y su objetivo fue el ampliar el espectro de usuarios de sus servicios educativos, más que la innovación. Los responsables de las EEV tienen diversas responsabilidades y sus competencias profesionales son multidisciplinarias. Ello le brinda una característica a las estructuras bajo su cargo: son activas promotoras de un modo de enseñar y aprender basado en las TIC y trabajan bajo los principios de aprendizaje, colaboración e innovación en contextos que rebasan continuamente las fronteras de la universidad. No existe un patrón dominante en la forma en que las EEV fueron creadas en la universidad, pero los casos en que los docentes innovadores tuvieron un papel importante, son los menos. Tampoco existe un tamaño de EEV preponderante, desde el punto de vista del número de personas que laboran en la misma.

En la caracterización del trabajo docente bajo modalidad de educación virtual, sobresalen ligeramente la práctica simultánea tanto de cursos habituales como de educación continua; la preponderancia de alguna forma de regulación institucional, la voluntariedad del profesorado y la ausencia de reconocimientos por el trabajo específico de educación virtual. Las dimensiones que empleamos en nuestro instrumento de trabajo: caracterización de los responsables de las EEV; caracterización del desarrollo de las mismas y condiciones básicas de trabajo de educación virtual en la universidad, nos permitieron corroborar algunas de las ideas básicas sobre implantación organizacional de la educación virtual en universidades que se presentaron en la Introducción. Se confirma el papel de la importancia del entorno; el liderazgo, la multidisciplinariedad y la idea de una trayectoria evolutiva de las estructuras.

Creemos que el tamaño de muestra y la dimensión latinoamericana de la misma representan una aportación a este tipo de estudios. La modalidad del nacimiento y desarrollo de las estructuras, así como las formas básicas de trabajo que los docentes tienen con la educación virtual, son aspectos que agregamos al conocimiento sobre el fenómeno. Queda claro que si hablamos del cambio organizacional en las universidades asociado a la adopción de la educación virtual, el fenómeno que se observa en las instituciones es el de una implantación de una estructura nueva dentro del conjunto de la universidad. Se trata de un órgano que emerge y se desarrolla articulando competencias tecnológicas con competencias profesionales y un contexto básico que le brinda la universidad de modo localizado.

\section{REFERENCIAS}

Alavi, M., D. Leinder, Virtual Learning Systems in Encyclopedia of Information Systems, vol. 4, Elsevier Science (2003)

Bates, A.W., Restructuring the University for Technological Change, in: Brennan, J., J. Fedrowitz, M. Huber, y T. Shah, eds., What kind of university? International perspectives on knowledge, participation and governance. Buckingham, The Society for Research into Higher Education \& Open University Press, 207-228 (1999) 
David ,P., D. Foray , Economic Fundamentals Of the Knowledge Society, SIEPR Discussion paper No. 01-14, Stanford, California, EUA (2002)

Doueihi, M., La gran conversión digital, Buenos Aires, Fondo de Cultura Económica (2010)

Drucker, P.F. Management, New York, Harper \& Row (1973)

Edel, R. , Las nuevas tecnologías para el aprendizaje: estado del arte, en Vales J. (ed.) Las nuevas tecnologías para el aprendizaje, México, Pearson- Prentice Hall (2009)

Esteve, J.M., La tercera revolución educativa. Una reflexión sobre nuestros profesores y nuestro sistema educativo en los inicios del siglo XXI. Contextos de educación (en línea) , 2001, http://www.unrc.edu.ar/publicar/cde/05/Esteve.htm, Acceso : 2 de junio (2011)

García, I., I. Peña-López, y cuatro autores más, Informe Horizon: Edición Iberoamericana 2010. Austin, Texas: The New Media Consortium (2010)

Garrison D.R y T. Anderson, El e-learning en el siglo XXI : Investigación y práctica, Barcelona, Octaedro (2005)

Hernández, R.; C. Fernández-Collado; P. Baptista, Metodología de la investigación, México, McGraw - Hill Interamericana, cuarta edición (2006)

Izcara, S.P., Introducción al muestreo; México, Miguel Angel Porrúa (2007)

Kalay, Y. E., Virtual Learning Environments , ITcon Vol. 9, Special Issue, pp. 195-207 (2004)

Kriger,T.J., A Virtual Revolution: Trends in the Expansion of Distance Education, USDLA Journal, ISSN 1537-5080 , en línea, Vol.15, No. 11,noviembre, 2001, http://www.usdla.org/html/journal/NOV01_Issue/article02.html,. Acceso: 15 de Julio (2011).

Micheli J. , Educación virtual y aprendizaje institucional México , Universidad Autónoma Metropolitana (2009)

Micheli J. y S. Armendáriz, Una tipología de la innovación organizacional para la educación virtual en universidades mexicanas, Revista de la Educación Superior, ANUIES, Volumen XXXIV (4), pp. 95-105 (2005)

Paulsen M.F., Experiences with Learning Management Systems in 113 European Institutions, Educational Technology \& Society , 6 (4) , 134-148, (2003)

Polsani, P., Use and Abuse of Reusable Learning Objects, Journal of Digital information, ISSN 1386-7506, en linea, 3(4). 2003, http://journals.tdl.org/jodi/article/viewArticle/89/88. Acceso: 12 de junio (2011)

Rama, C. (2010) La tendencia a la despresencialización de la educación superior en América Latina en RIED, Revista Iberoamericana de Educación a Distancia, Vol. 13, No. 1, pp. 39-72 (2010)

Rodriguez, J.L., La tercera función de la universidad In Micheli, J. (Coordinador), Educación virtual y aprendizaje institucional, pp. 22-32, México, Universidad Autónoma Metropolitana (2009)

Sancho Gil J., De tecnologías de la información y la comunicación a recursos educativos In Sancho Gil, J. (coordinadora), Tecnologías para transformar la educación, Universidad Internacional de Andalucía, AKAL (2006)

Serbin M. , The Wired Tower. Perspectives on the Impact of the Internet on Higher Education, New Jersey, Prentice Hall (2003)

Simone, R. La tercera fase. Formas de saber que estamos perdiendo, México,Taurus (2001) 
Singh, M. Universities and Society: Whose Term of Engagement? In Sörlin S. and H. Vessouri (editors) , Knowledge Society vs. Knowledge Economy: Knowledge; Power, and Politics, pp.53-78, New York, Palgrave Macmillan (2007)

Sörlin, S. y H. Vessuri, Introduction: The Democratic Deficit of Knowledge Economies, In Sörlin S. and $\mathrm{H}$. Vessouri (editors), Knowledge Society vs. Knowledge Economy: Knowledge; Power, and Politics, pp. 1-34, New York, Palgrave Macmillan (2007)

UNESCO, Hacia las sociedades del conocimiento, Paris (2005)

U.S. Department of Education Evaluation of Evidence-Based Practices in Online Learning (en línea) 2009. http://repository.alt.ac.uk/629/1/US_DepEdu_Final_report_2009.pdf,. Acceso: 15 de Julio (2011)

Wolff R., D. Roberts, A. Steed, O. Otto, A review of Tele- collaboration Technologies with Respect to Closely Coupled Collaboration, International Journal of Computer Applications in Technology , 29 (1) , pp.11-26 ( 2007) 